\title{
Anesthetized Intravenous Lethal Injection Euthanasia
}

National Cancer Institute

\section{Source}

National Cancer Institute. Anesthetized Intravenous Lethal Injection Euthanasia. NCI

Thesaurus. Code C116218.

A method of euthanasia whereby a subject is anesthetized and a lethal chemical is administered by intravenous injection to induce death. 